\title{
Searching for pulsars in future radio continuum surveys
}

\author{
Shi Dai ${ }^{1 *}$, Simon Johnston ${ }^{1}$ and George Hobbs ${ }^{1}$ \\ ${ }^{1}$ CSIRO Astronomy and Space Science, Australia Telescope National Facility, \\ PO Box 76, Epping, NSW, Australia \\ *email: shi.dai@csiro.au
}

\begin{abstract}
Radio continuum surveys are equally sensitive to all pulsars, not affected by dispersion measure smearing, scattering or orbital modulation of spin periods, and therefore allow us to search for extreme pulsars, such as sub-millisecond pulsars, pulsar-black hole systems and pulsars in the Galactic Centre. As we move towards the Square Kilometre Array (SKA) era, searching for pulsars in continuum images will complement conventional pulsar searches, and make it possible to find extreme objects.
\end{abstract}

Keywords. methods: observational - pulsars: general - radio continuum: general.

\section{Introduction}

The next-generation of radio continuum surveys will provide us with ultra-deep images of large swathes of the sky (e.g., Prandoni \& Seymour 2015). Millions of radio sources will be detected, a tiny (but significant) fraction of which will be radio pulsars. Radio continuum surveys have the potential to identify pulsars missed by the conventional techniques because the continuum imaging can detect pulsars independent of their pulse width. This is not the case with conventional pulsar searches which are most sensitive to narrow pulse-widths. A variety of effects can broaden the pulse profile, including dispersion-measure (DM) smearing, scattering and orbital modulation of spin periods. This makes it possible to uncover sub-millisecond pulsars, pulsar-blackhole systems and pulsars in the Galactic centre in continuum surveys.

\section{How to identify pulsars in continuum images?}

The main challenge of detecting pulsars in continuum surveys is to distinguish pulsars from other unresolved point-like radio sources. Continuum surveys such as the ASKAPEMU (Australian SKA Pathfinder-Evolutionary Map of the Universe; Norris et al. 2011) will identify $\sim 7 \times 10^{7}$ radio sources, while there are only $\sim 1.2 \times 10^{5}$ potentially observable pulsars in our Galaxy (e.g., Faucher-Giguère \& Kaspi 2006). Although pulsars are known to have steep spectra and high fractions of linear and circular polarisation, these features are not exclusive as galaxies can also have steep spectra and be highly polarised. However, pulsars are the only known sources compact enough to show diffractive interstellar scintillations (DISS), which distinguishes them from other radio sources.

DISS are observed as strong modulations of pulsar intensities caused by the scattering in the ionised interstellar medium. In Dai et al. (2016) we developed a formalism for computing variance images from standard, interferometric radio images containing time and frequency information. Such a technique can be applied on future continuum surveys to help us identify pulsars. As a demonstration, we used data of pulsar J0953+0755 taken 


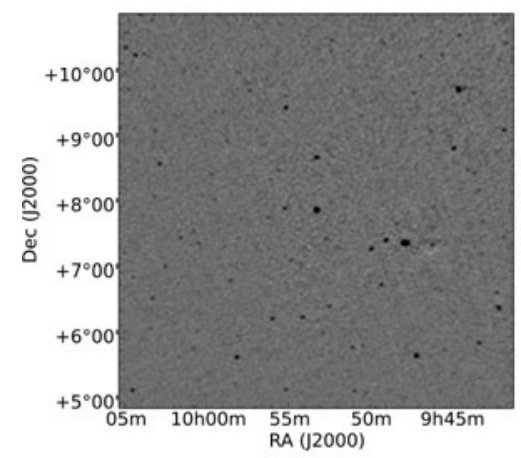

(a)

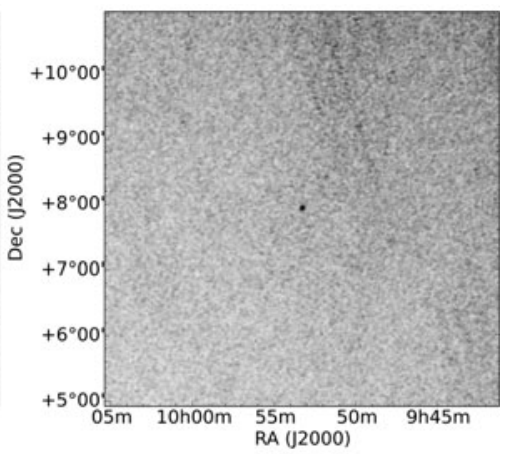

(b)

Figure 1. Demonstration of the variance imaging technique. (a) shows the Stokes I image with PSR J0953+0755 in the centre. (b) shows the variance image of the same field.

with the Murchison Widefield Array (MWA, Bell et al. 2016). Fig. 1 shows on the lefthand panel a continuum image made using the full $30.72 \mathrm{MHz}$ bandwidth. The right panel shows the variance image made with $1 \mathrm{MHz}$ channel bandwidth. In the continuum image, we can see a number of strong point-like radio sources, which are difficult to be distinguished from the pulsar without additional information. In contrast, in the variance image PSR J0953+0755 can be clearly identified and is the only source in the image.

\section{Detecting pulsars in variance images}

We carried out simulations to predict the number of pulsars that we can uncover with variance imaging on future continuum surveys (Dai et al. 2017). We show that the EMU survey can find $\sim 30$ normal pulsars and $\sim 40$ millisecond pulsars (MSPs) over and above the number known today, and similarly an all-sky continuum survey with SKA-MID can discover $\sim 140$ normal pulsars and $\sim 110$ MSPs with this technique. Variance imaging with EMU and SKA-MID will detect pulsars with large duty cycles and is therefore a potential tool for finding MSPs and pulsars in relativistic binary systems. Compared with current pulsar surveys at high Galactic latitudes in the southern hemisphere, variance imaging with EMU and SKA-MID will be more sensitive, and will enable detection of pulsars with dispersion measures between $\sim 10$ and $100 \mathrm{~cm}^{-3} \mathrm{pc}$.

Although variance imaging can help us distinguish pulsars from other compact radio sources, it is less sensitive than continuum imaging and is limited to relatively low DM pulsars. Variance imaging is only one possible avenue for finding pulsars in continuum images; searches in polarisation, rotation measure and circular polarisation are other options.

\section{References}

Bell, M. E., et al. 2016, MNRAS, 461, 908

Dai, S., et al. 2016, MNRAS, 462, 3115

Dai, S., Johnston, S., \& Hobbs, G. 2017, MNRAS, 472, 1458

Faucher-Giguère, C. \& Kaspi, V. M. 2006, ApJ, 643, 332

Norris, R. P., et al. 2011, PASA, 28, 215

Prandoni, I. \& Seymour, N. 2015, Advancing Astrophysics with the Square Kilometre Array (AASKA14), p. 67 